\title{
Dynamics of 4-(2-hexyloxy-ethoxy) 4'-cyanobiphenyl Molecules on Approaching a Glass Transition as Studied by Dielectric Spectroscopy
}

\author{
M. Massalska-Arodźt ${ }^{a *}$, W. Witko ${ }^{a}, \mathrm{~V} . \mathrm{GorbaCheV}^{b}$,
} L. HartmanN ${ }^{c}$ AND F. KREMER ${ }^{c}$

${ }^{a}$ The H. Niewodniczański Institute of Nuclear Physics

Polish Academy of Sciences, Radzikowskiego 152, 31-342 Kraków, Poland

${ }^{b}$ Department of Physical Chemistry, National Technical University of Ukraine 03-056 Kiev, Ukraine

${ }^{c}$ University of Leipzig, Department of Physics, 04103 Leipzig, Germany

(Received April 14, 2003; revised version September 15, 2003)

The complex dielectric permittivity of 4-(2-hexyloxy-ethyloxy) 4'-cyanobiphenyl measured in the frequency range from $0.03 \mathrm{~Hz}$ to $10^{9} \mathrm{~Hz}$ is presented. Monotropic sequence of thermodynamic phases was found with two stable phases (isotropic and crystal) and three metastable states (supercooled isotropic phase, its glass and a smectic phase observed only on heating the sample first cooled rapidly to the liquid nitrogen temperature). In isotropic and supercooled isotropic phases the temperature dependence of the dielectric relaxation time was described by the same Vogel-Fulcher-Tammann formula while in a smectic phase it was of the Arrhenius type. In the transition from a supercooled isotropic phase to a smectic phase the relaxation rate of molecular motions around the short axes increases by two decades. Comparison of the relaxation behaviour and polymorphism of 4-(2-hexyloxy-ethyloxy) 4'-cyanobiphenyl of linear molecules with two chiral cyanobiphenyls is discussed.

PACS numbers: $64.70 . \mathrm{Md}, 64.70 . \mathrm{Pf}, 77.22 . \mathrm{Gm}$

${ }^{*}$ corresponding author; e-mail: Maria.Masalska-Arodz@ifj.edu.pl 


\section{Introduction}

Derivatives of the cyanobiphenyl form a class of liquid crystalline compounds which has been studied dielectrically since the middle of seventies due to the fact that the strong dipolar moment of molecules is situated almost parallel to the long axes [1]. In higher members of the homologous series smectic phases were found [2]. Cyanobiphenyls having linear molecules indicate the reversible system of phase transitions and the Arrhenius type of relaxation. Recently two derivatives of cyanobiphenyls, namely optically active pentyl $\left(5^{*} \mathrm{CB}\right)$ and octyloxycyanobiphenyl ( $\left.8^{*} \mathrm{OCB}\right)$ were studied by several experimental methods [3-5]. Both were found to be fragile glass-formers [6] having monotropic sequence of phase transitions. The evidence of non-Arrhenius evolution of the so-called $\alpha$-relaxation on approaching glass transition has been given. It was specially interesting that crystallization was observed on heating the sample.

In the present paper the results of the dielectric relaxation studies in the 4-(2-hexyloxy-ethoxy) 4'-cyanobiphenyl $\mathrm{CH}_{3}\left(\mathrm{CH}_{2}\right)_{5} \mathrm{O}\left(\mathrm{CH}_{2}\right)_{2} \mathrm{O}\left(\mathrm{C}_{6} \mathrm{H}_{4}\right)_{2} \mathrm{CN}$, abbreviated as $6 \mathrm{O} 2 \mathrm{OCB}$, are presented. The molecules are non-chiral, however, their hexyloxy-ethoxy terminal chain $\left(\mathrm{C}_{6} \mathrm{H}_{13} \mathrm{OC}_{2} \mathrm{H}_{4} \mathrm{O}-\right)$ with two oxygen atoms has a complicated shape similarly as chiral chains of the previously studied compounds. It seems interesting to find out whether the relaxational behaviour and the polymorphism of $6 \mathrm{O} 2 \mathrm{OCB}$ with linear molecules resemble those of substances with chiral molecules of similar structure.

\section{Experimental details}

The sample was synthesized in Professor R. Dąbrowski laboratory at the Military University of Technology, Warsaw. It is a liquid at room temperature and crystallizes at $292 \mathrm{~K}$. The measurements of complex dielectric permittivity $\varepsilon^{*}(\nu)=\varepsilon^{\prime}(\nu)-\mathrm{i} \varepsilon^{\prime \prime}(\nu)$ were performed in the frequency range $\nu$ from $0.03 \mathrm{~Hz}$ to $1.8 \times 10^{9} \mathrm{~Hz}$. The value $\varepsilon^{\prime}$ means the real permittivity and $\varepsilon^{\prime \prime}$ is the dielectric loss. In measurements the Solartron FRA 1260 frequency response analyzer with the Novocontrol active sample cell BDC-S of $10^{-3}$ resolution of $\varepsilon^{\prime \prime}$ was used up to $10^{7} \mathrm{~Hz}$. In higher frequencies, from $10^{6} \mathrm{~Hz}$ to $1.8 \times 10^{9} \mathrm{~Hz}$, the Hewlett Packard $4291 \mathrm{~A}$ impedance analyzer with a resolution in $\tan \delta$ better than $10^{-2}$ was applied. In both ranges of frequency the liquid sample was placed between two brass electrodes of effective diameter $1 \mathrm{~cm}$ and $0.5 \mathrm{~cm}$ (for higher frequencies) with $0.05 \mathrm{~mm}$ distance between them kept constant using several teflon strips. The studies were performed on heating the sample (after rapid cooling to $100 \mathrm{~K}$ with cooling rate of $100 \mathrm{~K} / \mathrm{min}$ ) in the temperature range from $200 \mathrm{~K}$ to room temperature at $2.5 \mathrm{~K}$ intervals with accuracy of $0.1 \mathrm{~K}$. The sample temperature was controlled using a Novocontrol Quatro system with a liquid nitrogen cryostat. In chosen temperature ranges, the measurements on cooling were also performed. The rate of temperature changes during measurements was of about $0.3 \mathrm{~K} / \mathrm{min}$. 


\section{Results}

Several temperature runs were performed to check the sequence of phase transitions in $6 \mathrm{O} 2 \mathrm{OCB}$. On slow cooling the sample $(0.3 \mathrm{~K} / \mathrm{min})$ the crystallization was always observed at $292 \mathrm{~K}$. When the sample was first cooled rapidly to $100 \mathrm{~K}$ (with the cooling rate of about $100 \mathrm{~K} / \mathrm{min}$ ) a glass $(\mathrm{G})$ was obtained. Then on heating with a rate of $20 \mathrm{~K} / \mathrm{min}$ a softening of the glass to the supercooled isotropic phase $\left(\mathrm{I}_{\mathrm{sc}}\right)$ occurs. On further heating with a rate of $0.3 \mathrm{~K} / \mathrm{min}$ several phase transitions were found. They are shown in Fig. 1 by the temperature changes of

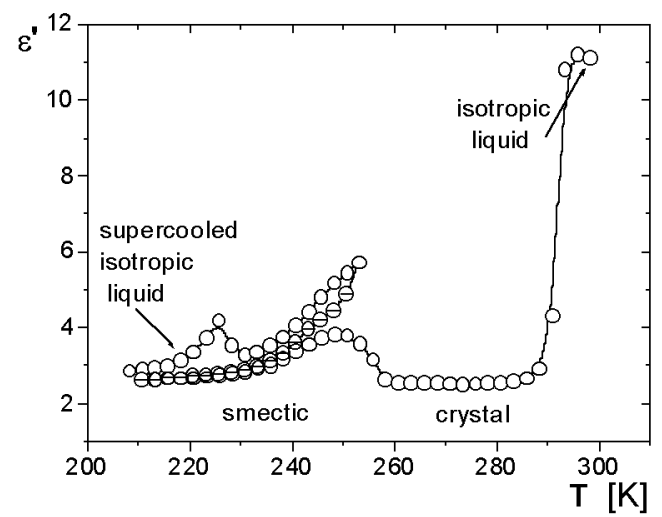

Fig. 1. Temperature changes of real dielectric permittivity observed at frequency $120 \mathrm{~Hz}$ on heating the sample up to $253 \mathrm{~K}(\mathrm{o})$ then on cooling to $210 \mathrm{~K}(\ominus)$ and on further heating up to $300 \mathrm{~K}(\mathrm{o})$. The experimental run illustrates the monotropic sequence of phases for $6 \mathrm{O} 20 \mathrm{CB}$. Measurements were performed with the rate of temperature changes of $0.3 \mathrm{~K}$.

electric permittivity for a chosen frequency of $120 \mathrm{~Hz}$. The phase sequence seems to be the following:

$$
\mathrm{G} \longrightarrow \mathrm{I}_{\mathrm{sc}} \stackrel{228 \mathrm{~K}}{\longrightarrow} \mathrm{S} \stackrel{260 \mathrm{~K}}{\longrightarrow} \mathrm{C} \stackrel{292 \mathrm{~K}}{\longrightarrow} \mathrm{I},
$$

where $\mathrm{S}$ means a smectic phase, $\mathrm{C}$ - a crystal and finally $\mathrm{I}$ - the stable isotropic phase. It was found that when heating was stopped at $253 \mathrm{~K}$ then on immediate cooling no phase transition was detected at all till $200 \mathrm{~K}$ and on subsequent heating the crystallization and then the melting were found. It means that the transition from $\mathrm{I}_{\mathrm{sc}}$ to $\mathrm{S}$ is irreversible and already at $253 \mathrm{~K}$ there are nuclei of the crystalline phase in the sample.

Figure 2 presents the frequency changes of dielectric absorption for $6 \mathrm{O} 2 \mathrm{OCB}$ in the frequency range from $0.03 \mathrm{~Hz}$ to $10^{9} \mathrm{~Hz}$ on heating the sample, which previously had been cooled rapidly to $100 \mathrm{~K}$, i.e. to glass. In Fig. 3a and b additionally the real and imaginary parts of permittivity are shown in a form of the three-dimensional plots in frequencies below $3 \mathrm{MHz}$. Observing the temperature 


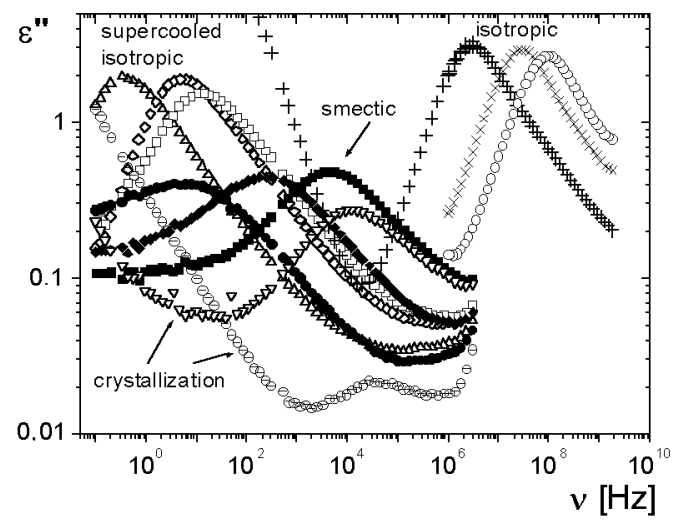

Fig. 2. Dielectric losses vs. frequency observed for several temperatures on heating $6 \mathrm{O} 2 \mathrm{OCB}$ sample with the heating rate of $0.3 \mathrm{~K} / \mathrm{min}$. The data are presented in the double logarithmic scale. Meaning of the symbols in supercooled liquid: $\Delta-219 \mathrm{~K}$, $\diamond-224 \mathrm{~K}$ and $\square-226 \mathrm{~K}$, in the smectic phase: $\bullet-234 \mathrm{~K}, \boldsymbol{\wedge}-245 \mathrm{~K}$ and $\mathbf{\boldsymbol { \imath }}-255$ $\mathrm{K}$, for the crystallization process: $\nabla-257 \mathrm{~K}$ and $\ominus-260 \mathrm{~K}$ and in the isotropic phase: $+-296 \mathrm{~K}, \times-330 \mathrm{~K}$ and $\mathrm{O}-360 \mathrm{~K}$. The data were collected by two apparatuses operating in two frequency ranges.
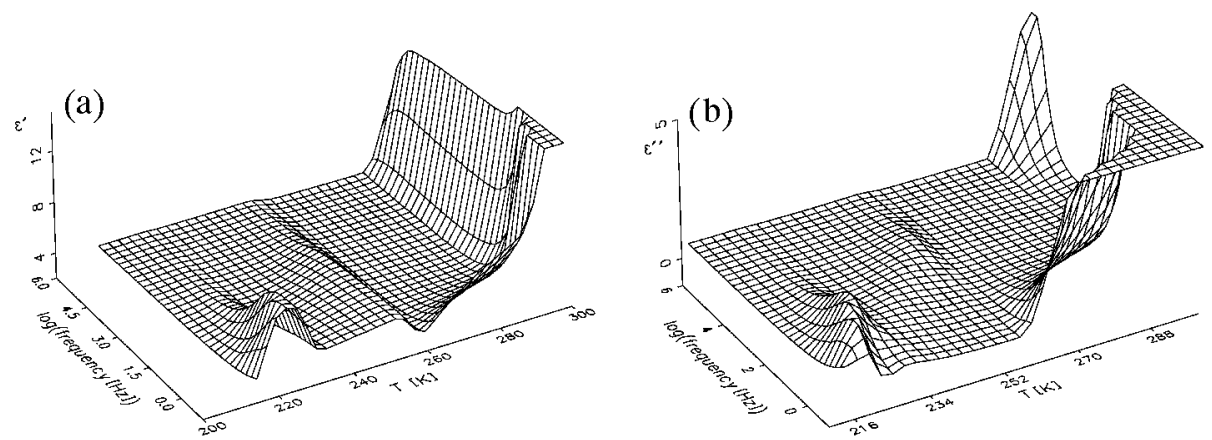

Fig. 3. (a) Real dielectric permittivity vs. frequency and vs. temperature observed on heating previously rapidly cooled $6 \mathrm{O} 2 \mathrm{OCB}$ sample (cooling rate $200 \mathrm{~K} / \mathrm{min}$ ) is presented for clarity at temperatures below $300 \mathrm{~K}$ and in the frequency range up to $3 \mathrm{MHz}$. Three dispersion ranges are shown: first in the supercooled isotropic phase (below $228 \mathrm{~K}$ ), second in the smectic phase (between $228 \mathrm{~K}$ and $260 \mathrm{~K}$ ) and the third in the isotropic phases (after melting at $292 \mathrm{~K}$ ). At about $260 \mathrm{~K}$ the crystallization is presented. Measurements were performed with the rate of temperature changes of $0.3 \mathrm{~K}$. A part of the electric conductivity at high temperatures and low frequencies is cut-off. (b) Dielectric loss vs. frequency and vs. temperature is presented. The thermal treatment of the sample and the temperature and frequency ranges are the same as for the corresponding real dielectric permittivity results in Fig. 3a. A part of the absorption connected with the electric conductivity at high temperatures and low frequencies is cut-off. 
changes of $\varepsilon^{\prime}$ and the dielectric absorption strength one can find the evidence of three phase transitions. A significant drop of $\varepsilon^{\prime \prime}$ and a shift of position $\nu_{\max }$ of absorption peak towards lower frequencies found during transition at about $228 \mathrm{~K}$ are presented in double logarithmic scale in Fig. 4. These results support the hypothesis that the sample transforms from the supercooled isotropic phase $\left(\mathrm{I}_{\mathrm{sc}}\right)$ to the smectic phase (S). Most likely arrangement of molecules in smectic layers di-

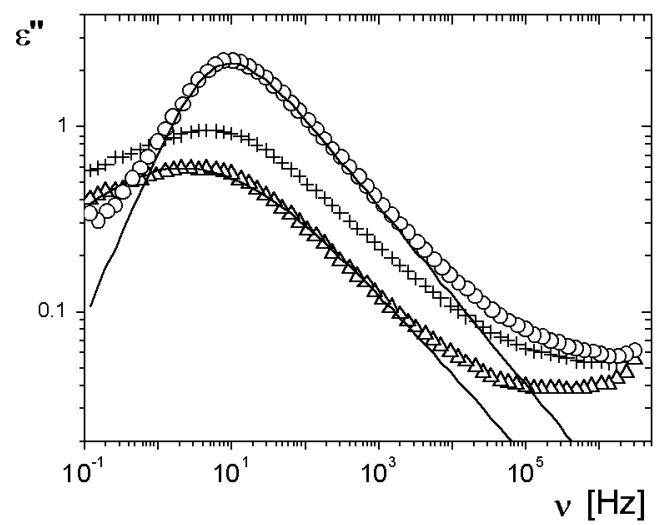

Fig. 4. Temperature changes of dielectric absorption in the vicinity of the isotropic to the smectic transition observed on heating of $6 \mathrm{O} 2 \mathrm{OCB}$ sample $(\mathrm{O}-223 \mathrm{~K}$ in the isotropic phase, $x-228 \mathrm{~K}$ for the mixture of the isotropic and smectic phases and $\Delta-233 \mathrm{~K}$ in the smectic phase). Data are presented in the double logarithmic scale. The results of fitting Eq. (1) are presented by the solid lines.

minishes the effective dipolar moment of reorienting molecules ( $\varepsilon_{\max }^{\prime \prime}$ decreases by factor 6) and slows molecular motions by factor 100 (when approximating to the same temperature) ${ }^{\dagger}$. On further heating the sample in a temperature range from $257 \mathrm{~K}$ to $261 \mathrm{~K}$ the decay of $\varepsilon^{\prime \prime}(\nu)$ absorption curves characteristic of the crystallization can be seen; at $260 \mathrm{~K}$ the strength of absorption is very small and the electric conductivity below $100 \mathrm{~Hz}$ was observed mainly. In a temperature range between $261 \mathrm{~K}$ and $290 \mathrm{~K}$ there is no dielectric relaxation at all which means that all molecules have lost their mobility. The melting of the crystal starts at $290 \mathrm{~K}$ which is shown in Fig. 5. One can see that both processes, i.e., the crystallization and the melting are not instantaneous and each lasts of about 50 minutes (between points measured at the successive temperatures there is about 20 minutes

${ }^{\dagger}$ The permittivity and dielectric absorption values in the smectic phase cannot be treated as the material constants as their values are not the same for each experimental run $\left(\varepsilon_{\max }^{\prime \prime}(\nu)\right.$ varies in the range from 0.3 to 0.8 ). This can be explained by the fact that external alignment of molecules in the smectic phase has not been possible as $6 \mathrm{O} 2 \mathrm{OCB}$ does not form the nematic phase [7]. Important is that it does not disturb interpretation of polymorphism and the molecular motions. 


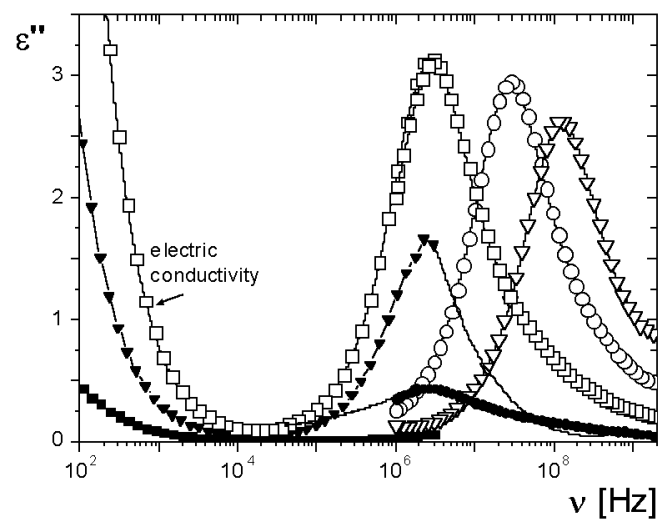

Fig. 5. Dielectric losses observed for several temperatures on heating the crystalline $6 \mathrm{O} 2 \mathrm{OCB}$ sample - data are from two apparatuses operating in different frequency ranges. Filled symbols illustrate $\varepsilon^{\prime \prime}(\nu)$ for crystal $(\mathbf{a}-290 \mathrm{~K})$ and for crystal during melting $(\bullet-291 \mathrm{~K}, \mathbf{T}-292 \mathrm{~K})$ while open symbols show the dielectric absorption in the isotropic phase $(\square-295 \mathrm{~K}, \circ-330 \mathrm{~K}$ and $\nabla-370 \mathrm{~K})$. Lines are the guides to the eyes only. The solid lines without symbols were calculated using the symmetrical reflection of the suitable experimental points with respect to $\nu_{\max }$.

for temperature stabilization). Long lasting recovery of the molecular mobility of the isotropic phase suggests that the melting process is complex.

Taking into account the structure of the 6O2OCB molecules the dielectric relaxation observed in isotropic liquid and in smectic phase can be related with reorientations of $\mathrm{CN}$ dipoles around the short axes. The experimental results of $\varepsilon^{\prime \prime}(\nu)$ are divided into electric conductivity and dielectric relaxation according to the Havriliak-Negami equation [8]

$$
\varepsilon^{\prime \prime}(\nu)=\frac{\sigma_{0}}{2 \varepsilon \pi \nu}-\operatorname{Im}\left[\Delta \varepsilon\left(1+(2 \mathrm{i} \pi \nu \tau)^{b}\right)^{-g}\right],
$$

where $\sigma_{0}$ means the electric conductivity, $\varepsilon$ - dielectric constant of vacuum and $\tau=1 /(2 \pi \nu)$ is the average relaxation time. The dielectric increment $\Delta \varepsilon=\varepsilon_{0}-\varepsilon_{\infty}$, where $\varepsilon_{0}$ and $\varepsilon_{\infty}$ denote the static and high frequency limits of electric permittivity, respectively. Relaxation time distribution parameters $b$ and $b g$ fulfill the relations $0 \leq b \leq 1$ and $0 \leq b g \leq 1$ and they are defined by the following equations (see $[8,9])$ :

$$
\text { for } \nu \ll \nu_{\max } \quad b=\frac{d \log \varepsilon^{\prime \prime}}{d \log 2 \pi \nu} \quad \text { and for } \nu \gg \nu_{\max } \quad b g=\frac{d \log \varepsilon^{\prime \prime}}{d \log 2 \pi \nu} .
$$

The relations between the Havriliak-Negami distribution parameters and those of the Cole-Cole and Cole-Davidson formulae are the following: $\alpha_{\mathrm{C}-\mathrm{C}}=b$ for $g=1$ and $\beta_{\mathrm{C}-\mathrm{D}}=g$ for $b=1$. For the Debye type of relaxation both $b=1$ and $g=1$. The $\Delta \varepsilon, b, g$, the half-width $\Delta \nu_{1 / 2}$ and the respective values of relaxation time were obtained by fitting the formula (1) to the experimental data - two of the 
examples of the fitting results are presented in Fig. 4. In the isotropic phase there is $b=1$, while $b g$ grows with temperature from 0.4 to 0.8 . Thus, the relaxation is of the Cole-Davidson type. The values of the half-width $\Delta \nu_{1 / 2}$ decrease from 1.6 to 1.3 with increasing temperature so they are larger than $\Delta \nu_{1 / 2}=1.14$ typical of the Debye relaxation. In the smectic phase the distribution parameters are $b \sim 0.4$ and $b g \sim 0.5$, and the half-width is over 3 which suggests a large deviation from the Debye process. That fact can be ascribed to the strong hindrance of molecular motions around the short axes in the smectic type arrangement of molecules. One can see that for proper description of the dielectric relaxation both $b$ and $g$ parameters are needed especially in the smectic phase.

The dielectric increment, $\Delta \varepsilon(T)=f N \mu^{2} /(3 k T)$, is proportional to the number of reorienting dipoles $N$, to $\mu^{2}$, where $\mu \sim 4 \mathrm{D}$ is the dipolar moment of molecule, to the so-called Kirkwood correlation factor $f$ and it is reciprocal to the temperature [10]. $\Delta \varepsilon$ equals nearly 6 in the isotropic phase and nearly 2 in the smectic phase. In case when in the isotropic-smectic phase transition the $N$ value does not change one can say that $f$ in the smectic phase is 3 times smaller than in the isotropic phase close to the temperature of phase transition. Assuming further weak mutual correlations between molecules in the isotropic phase, i.e. $f_{\text {is }} \approx 1$, one obtains $f_{\text {aniz }} \approx 0.33$. The estimation of the correlation factor in the smectic phase with help of the Kirkwood-Fröhlich relationship taking into account additionally the local field factor gives exactly the same result [10]. That means the strong antiparallel correlations between molecules arranged in the smectic phase. One can see that the $\Delta \varepsilon$ diminishes in the smectic phase with decreasing temperature which supports the above statement.

Figure 6 presents the temperature dependence of the position of the dielectric absorption maximum observed on heating for the sample cooled at first rapidly to $100 \mathrm{~K}$. Unusual pattern of the $\log \left(2 \pi \nu_{\max }\right)$ vs. $1000 / T$ results confirms that on cooling the sample to $100 \mathrm{~K}$ a glass of the isotropic phase was obtained: One can see that the relaxation in the supercooled isotropic phase (four points) and in the isotropic phase can be commonly described by the same Vogel-Fulcher-Tammann equation [11] typical for glass-formers

$$
\tau=\tau_{0} \exp \frac{D T_{0}}{\left(T-T_{0}\right)}
$$

It is especially interesting because between the supercooled isotropic liquid and the isotropic phase there are two other thermodynamic phases, smectic and crystalline. At $228 \mathrm{~K}$ the maximum absorption frequency diminishes abruptly due to the transition to the smectic phase. At $260 \mathrm{~K}$ the crystallization occurs and there are no experimental points up to the melting at about $292 \mathrm{~K}$. The best fit of (2) to the experimental data (solid line) was obtained for the following fitting parameters: $D=3.6$ and $T_{0}=167 \mathrm{~K}$, where $T_{0}$ denotes the lowest temperature at which a glass can be obtained. The value of $\tau_{0}$ has been fixed as $10^{-13.5} \mathrm{~s}$ in order to relate the $D$ parameter with the fragility introduced by Angell for description of 


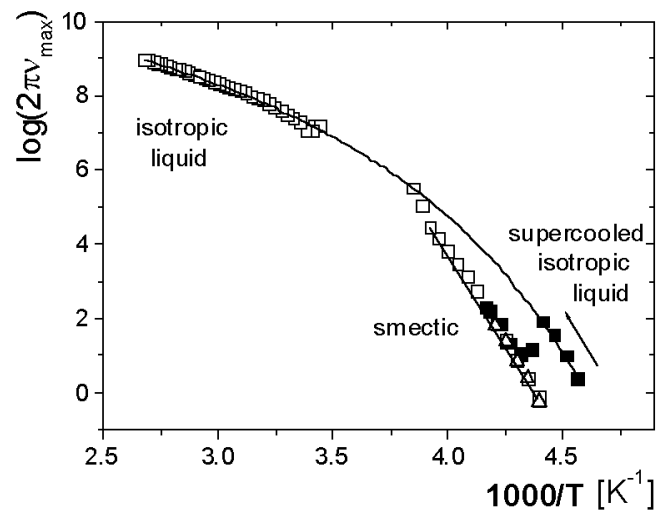

Fig. 6. The temperature changes of dielectric relaxation for $602 \mathrm{OCB}$ sample presented by $\log \left(2 \pi \nu_{\max }\right)$ vs. $1000 / T$ for the temperature run are shown. Data were collected on heating ( $\mathbf{a})$ then on cooling $(\Delta)$ and on further heating ( $\square$ ). The result of fitting the formula (2) to the data for the isotropic and supercooled isotropic phase is shown by the solid line. The lack of points in the temperature range from $260 \mathrm{~K}$ to $292 \mathrm{~K}$ means the appearance of the crystalline phase. Straight lines show the fitting of the Arrhenius formula.

the $\alpha$-relaxation $[12,13] . D$ value is not far from $D=3$ which is the limit value for the most fragile glasses ${ }^{\ddagger}$. It seems worth to note that at $228 \mathrm{~K}$ the relaxation time in the smectic phase is 2 decades longer than in the supercooled isotropic phase. Within the smectic phase the relaxation was observed also on cooling the sample. The $\log \left[2 \pi \nu_{\max }(1 / T)\right]$ points obtained on cooling and on heating are in good agreement. One can see that the temperature behaviour of the molecular dynamic in the isotropic phase and in the smectic phase is different: in the smectic phase the linear Arrhenius temperature dependence of relaxation is observed giving activation energy $\Delta H=87.7 \mathrm{~kJ} / \mathrm{mol}$.

\section{Final remarks}

Our dielectric relaxation observations show that there are essential differences between $6 \mathrm{O} 2 \mathrm{OCB}$ and two chiral substances with a similar shape of molecules in respect to the crystallization phenomenon. Contrary to $5^{*} \mathrm{CB}$ and $8^{*} \mathrm{OCB}$ the $6 \mathrm{O} 2 \mathrm{OCB}$ is not a glass-former: For $6 \mathrm{O} 2 \mathrm{OCB}$ the crystallization is observed on cooling while $5^{*} \mathrm{CB}$ and $8^{*} \mathrm{OCB}$ have crystallized only on heating the sample because on cooling a glass was always obtained [3-5].

In the case when $6 \mathrm{O} 2 \mathrm{OCB}$ sample was cooled rapidly $(\sim 100 \mathrm{~K} / \mathrm{min})$ to glass a polymorphism of $6 \mathrm{O} 2 \mathrm{OCB}$ and that of the chiral compounds have had common

\footnotetext{
$\ddagger$ Fragility of the $\alpha$-relaxation is considered as a measure of generation of the configurational entropy in the liquid $[9,6]$. A higher fragility is manifested by the larger deviation of the relaxation from the Arrhenius type of temperature dependence.
} 
features: The phase transitions are irreversible and form monotropic sequence of phases. Non-chirality of $6 \mathrm{O} 2 \mathrm{OCB}$ molecules seems to be the factor which helps a creation of the smectic ordering which was unobserved for chiral samples. The observed texture of this anisotropic phase is similar to that of ordered smectic phases. To determine the full structure of this smectic phase more detailed studies must be undertaken. Due to the fact that the crystallization observed for $6 \mathrm{O} 2 \mathrm{OCB}$ sample is a long lasting process, one can speculate that after it melts at about $292 \mathrm{~K}$, immediately the second crystalline phase is being formed which melts at a slightly higher temperature $[4,5]$. Detailed studies by polarizing microscopy and calorimetry were undertaken to search the second crystalline phase in 6O2OCB [14] as it was found for $5^{*} \mathrm{CB}$ and $8^{*} \mathrm{OCB}[3-5]$.

In three substances which have been compared the dielectric relaxation in the liquid phase fulfils the Vogel-Fulcher-Tammann equation in a very wide temperature range. It is interesting that nearly $200 \mathrm{~K}$ above $T_{0}$ the rate of molecular motions behaves in isotropic liquid like in a glass-former even for 6O2OCB which in fact is the substance where glass can be obtained only when the rate of cooling is high. The $6 \mathrm{O} 2 \mathrm{OCB}$ sample having $D=3.6$ occurs to be a more fragile glass-former than $5^{*} \mathrm{CB}$ and $8^{*} \mathrm{OCB}$ for which $D \cong 5.5[3]$. Analyzing the temperature dependence of relaxation rate carefully one can see that similarly as for $5^{*} \mathrm{CB}[4]$ the crystallization seems to be proceeded by the melting of the smectic phase to the isotropic phase. This can be deduced from the deviation of the experimental points in Fig. 6 in the vicinity of the crystallization from the activation behaviour (see two points above the streight line).

\section{Acknowledgments}

This work was partially supported by grant No. 2 P03B 02618 of the State Committee for Scientific Research. One of us (M.M.-A.) would like to thank the Leipzig University for financial support enabling the measurements at Leipzig University.

\section{References}

[1] M. Davies, R. Moutran, A.H. Price, M.S. Beevers, G. Williams, J. Chem. Soc. Faraday, Trans. 72, 1447 (1976); D. Lippens, J.P. Parneix, A. Chapoton, J. Phys. (France) 38, 1465 (1977); B.R. Ratna, R. Shashidar, Mol. Cryst. Liq. Cryst. 42, 185 (1977); J.M. Wacrenier, C. Druon, D. Lippens, Mol. Phys. 43, 97 (1981); S. Urban, B. Gestblom, A. Wurflinger, Mol. Cryst. Liq. Cryst. 331, 113 (1999) and the references therein.

[2] G.W. Gray, J.W.G. Goodby, Smectic Liquid Crystals. Textures and Structure, Leonard Hill, Glasgow 1984, p. 6; P.E. Cladis, R.K. Bogardus, D. Aadsen, Phys. Rev. A. 18, 2292 (1978). 
[3] M. Massalska-Arodź, G. Williams, I. Smith, Ch. Conolly, G.A. Aldridge, R. Dąbrowski, J. Chem. Soc. Faraday Trans. 94, 387 (1998); M. Massalska-Arodź, G. Williams, D. Thomas, J. Jones, R. Dąbrowski, J. Phys. Chem. B 103, 4197 (1999).

[4] J. Mayer, W. Witko, M. Massalska-Arodź, G. Williams, R. Dąbrowski, Phase Transit. 69, 199 (1999); W. Witko, J. Ściesiński, E. Ściesińska, M. Massalska-Arodź, J. Mayer, R. Dąbrowski, Mol. Cryst. Liq. Cryst. 330, 391 (1999); S. Urban, B. Gestblom, R. Dąbrowski, Phys. Chem. Chem. Phys. 1, 4843 (1999).

[5] J. Ściesiński, E. Ściesińska, M. Massalska-Arodź, T. Wasiutyński, P.M. Zieliński, W. Witko, IEEE Trans. Dielectr. Electr. Insul. 8, 522 (2001).

[6] R. Bohmer, K.L. Ngai, C.A. Angell, D.L. Plazek, J. Chem. Phys. 99, 4201 (1993).

[7] M. Massalska-Arodź, H. Schmalfuss, W. Witko, H. Kresse, A. Wurflinger, Mol. Cryst. Liq. Cryst. 364, 221 (2001).

[8] S. Havriliak, D.G. Watts, Polymer 27, 1509 (1986); S.J. Havriliak Jr., S.J. Havriliak, J. Non-Cryst. Solids 172-174, 297 (1994).

[9] A.P. Donth, Glass Transition, Springer-Verlag, Berlin 2001.

[10] C.J.F. Bottcher, P. Bordewijk, Theory of Electric Polarization, Vol. II, Elsevier Scientific, Amsterdam 1978.

[11] H. Vogel, Phys. Z. 22, 645 (1921); G.S. Fulcher, J. Am. Ceram. Soc. 8, 339 (1923); M.H. Cohen, G.S. Grest, Phys. Rev. B 20, 1077 (1979).

[12] C.A. Angell, J. Non-Cryst. Solids 131-133, 13 (1991); C.A. Angell, Polymer 38, 4201 (1993).

[13] L.M. Torell, L. Borjesson, M. Elmroth, J. Phys., Condens. Matter 2, 205 (1990).

[14] W. Witko, J. Mayer, unpublished. 